\title{
Les institutions et les médias
}

De l'analyse du discours à la traduction

\section{Le istituzioni e i media \\ Dall'analisi del discorso alla traduzione}

Édité par

Marie-Christine Jullion, Louis-Marie Clouet et Ilaria Cennamo 
Copyright (C) 2019

$\mathcal{} \perp$ Edizioni Universitarie di $\mathcal{L}_{\text {ettere }} E_{\text {conomia }}$ Diritto

Via Cervignano 4 - 20137 Milano

www.lededizioni.com - www.ledonline.it - E-mail: led@lededizioni.com

I diritti di riproduzione, memorizzazione e archiviazione elettronica, pubblicazione con qualsiasi mezzo analogico o digitale

(comprese le copie fotostatiche, i supporti digitali e l'inserimento in banche dati) e i diritti di traduzione e di adattamento totale o parziale sono riservati per tutti i paesi.

Le fotocopie per uso personale del lettore possono essere effettuate nei limiti del 15\% di ciascun volume/fascicolo di periodico dietro pagamento alla SIAE del compenso previsto dall'art. 68, commi 4 e 5, della legge 22 aprile 1941 n. 633.

Le riproduzioni effettuate per finalità di carattere professionale, economico o commerciale o comunque per uso diverso da quello personale possono essere effettuate a seguito di specifica autorizzazione rilasciata da: AIDRO, Corso di Porta Romana n. 108 - 20122 Milano E-mail segreteria@aidro.org <mailto:segreteria@aidro.org> sito web www.aidro.org <http://www.aidro.org/>

\author{
Volume stampato con il contributo \\ del Dipartimento di Scienze della Mediazione Linguistica e di Studi Interculturali \\ Università degli Studi di Milano
}

Videoimpaginazione: Paola Mignanego

Stampa: Logo 


\title{
Table des matières
}

\author{
INTRODUCTION
}

Les institutions et les médias: un univers de discours et de traductions Marie-Christine Jullion - Louis-Marie Clouet - Ilaria Cennamo

Les conditions de l'analyse du discours pour l'étude des débats publics Patrick Charaudeau

La gestion des identités dans certains types de débats médiatiques: le rôle des termes d'adresse

Catherine Kerbrat-Orecchioni

De l'analyse de discours à la traduction: la médiation interculturelle

Christine Durieux

Scrivere chiaro per tradurre chiara mente

Antonella Leoncini Bartoli

Towards a Linguistic Definition of 'Simplified Medical English':

Applying Textometric Analysis to Cochrane Medical Abstracts and Their Plain Language Versions

Christopher Gledhill - Hanna Martikainen - Alexandra Mestivier

Maria Zimina

Traduction de la 'qualité', qualité de la traduction: une analyse

des traductions française et italienne des ESG Standards and Guidelines Micaela Rossi

Simultaneous Interpretation of Political Discourse:

Coping Strategies vs Discourse Strategies. A Case Study

Alicja M. Okoniewska

Tradurre il discorso istituzionale pubblico: una riflessione sulla traduzione come mediazione interculturale Ilaria Cennamo

“让中华文化展现出永久魅力和时代风采 Que la beauté éternelle

de la culture chinoise rayonne sur notre époque": la terminologie de la culture dans le discours au XIX ${ }^{\mathrm{e}}$ Congrès du Parti Communiste Chinois

Pascale Elbaz 
Il discorso polemico politico e la formula nel dibattito politico sui media e sulle reti sociali: il caso del 'mariage pour tous' Daniela Virone

Filo da torcere: percezione e trattamento del discorso politico italiano in cabina d'interpretazione Ludovica Maggi

La traduction du gérondif et du participe présent dans un corpus parallèle de textes parlementaires européens: réflexions traductologiques

Stéphane Patin

L'analisi delle trascrizioni di colloqui medici nella didattica dell'interpretazione telefonica Nora Gattiglia

Le terme ğibād: un malentendu dans le discours médiatique Malek al-Zaum

L'Ayuntamiento de Madrid in Twitter: un'analisi linguistico-discorsiva Giovanna Mapelli 


\title{
Les conditions de l'analyse du discours pour l'étude des débats publics*
}

\author{
Patrick Charaudeau
}

DOI: http://dx.doi.org/10.7359/919-2019-char

\begin{abstract}
Public debate is essentially a matter of language. It implies two or more interlocutors who discuss, explain, tell and argue about some issues. Actually, discourse processes depend on the context of communication as well as on the relational distance between interlocutors. This is why different kinds of communicative exchange can be established according to precise criteria, thus determining the existence of different categories of debates. By analysing those differences, it is possible to distinguish between what is called "controversy" with respect to "polemic", two concepts that are often confused.
\end{abstract}

Mots-clés: controverse; débat; échange interlocutif; polémique; typologie.

Keywords: controversy; debate; interlocutory exchange; polemic; typology.

\section{INTRODUCTION}

Le débat public peut être l'objet d'étude de diverses disciplines des sciences humaines et sociales. Mais il est évident qu'il est un objet central pour les sciences du langage, le débat étant un lieu d'entrecroisement de paroles. Cependant, à l'intérieur de ce domaine disciplinaire, il y a diverses façons d'analyser les faits de langage, selon diverses approches: textuelles, pragmatiques, interactionnistes, sociolinguistiques, sociodiscursives, etc., et selon des procédures quantitatives ou qualitatives. On ne va pas ici passer en revue ces différentes théories et méthodes, mais seu-

* J'ai longuement analysé cette question dans le livre Charaudeau 2017. C'est pourquoi, je serai amené à en reprendre certains passages. 
lement lever un malentendu sur la façon d'analyser les faits de langage, certaines disciplines pratiquant ce que l'on appelle l'analyse de contenu. Or, celle-ci n'est pas en tout point identique à l'analyse de discours. La première considère le matériau langagier comme un donné, explicitement repérable. Elle traite la matérialité du langage dans sa manifestation explicite, ce qui lui permet de procéder à une segmentation et un classement du dit en un certain nombre de thèmes. Cette catégorisation thématique peut, ainsi, être traitée de façon quantitative ${ }^{1}$.

L'analyse du discours, elle, considère le matériau langagier comme un processus dont le sens se construit dans l'acte même de production de la parole. Le sens qui en résulte est opaque et pluriel, se trouvant dans le non dit, l'implicite, un implicite qui entre en conflit avec l'explicite. Le sens de tout acte de langage n'est pas seulement dans "ce que l'on dit", mais dans "comment on le dit". Ce qui fait que l'analyse du discours oblige à tenir compte des conditions de productions de l'acte de langage: conditions situationnelles (qui parle à qui, dans quelle circonstance, selon quel enjeu); conditions énonciatives (quel type de mise en scène du langage, entre le Je-locuteur qui prend la parole, le Je-énonciateur qui énonce, le Tu-destinataire auquel il s'adresse); conditions rhétoriques (organisation phrastique, organisation de la textualité, jeu lexical, organisations descriptive, narrative et argumentative). De plus, il faut tenir compte du fait que le sens construit par le sujet parlant n'est pas susceptible de correspondre à celui que construit le récepteur lors de son interprétation, autrement dit que l'effet visé (effet intentionnel du locuteur) ne correspond pas en tout point à l'effet produit sur le récepteur.

C'est donc en fonction de ces conditions de production générales que je vais décrire ce que sont les conditions propres à l'analyse des débats puisqu'on se trouve ici dans le cas particulier d'actes de langage produits en situation d'interactions verbales.

\section{LES CONDITIONS D'ANALYSE D’UN DÉBAT}

Un débat étant un processus d'échange de paroles, il convient, dans un premier temps, de déterminer les critères selon lesquels peuvent être analysés ces échanges. Cela permettra ensuite de tenter de distinguer divers types d'échange, autrement dit d'ébaucher une typologie des échanges. Cette légitimité repose à la fois sur la qualité sociale de l'individu qui parle et de ce au nom de quoi il parle.

1 D’où la concurrence entre divers logiciels de traitement des données (lexicométrie). 
- Le statut langagier - Il s'agit d'un statut proprement langagier, c'est-àdire en relation avec ce qui détermine le droit à la prise de parole, ce qui légitime le sujet en tant qu'être communiquant. Un médecin sera légitimé, par son statut socio-professionnel dans le cabinet de consultation, ce qui lui permettra de poser certaines questions intimes à son patient, mais ailleurs, ce sera en tant qu'ami, voisin de palier ou client d'un restaurant, et, dans chacune de ces situations, il parlera au nom de ce qui est en jeu dans l'échange. Le statut langagier permet de déterminer le type de rapport qui existe entre les participants à l'échange: rapport d'égalité, rapport de hiérarchie, de supériorité ou d'infériorité, ou tout simplement rapport de différence.

- La thématique - La nature du sujet traité par les participants à l'échange engage un certain type de savoir. Ce savoir peut être plus ou moins spécialisé: un savoir de connaissance, comme on le verrait mis en œuvre dans les débats scientifiques, un savoir de croyance, comme dans les débats doctrinaux, un savoir d'opinion comme dans les débats de société. On peut ainsi observer si le savoir est partagé entre les participants, ou prendre acte des disparités. Ce critère permettra, entre autres, de distinguer des types de controverse, comme on le verra plus loin.

- Le positionnement des interlocuteurs - En relation avec ces statuts et ces rôles, les participants de l'échange adoptent un certain positionnement qui instaure un rapport de complémentarité ou de symétrie entre eux. Un double positionnement: par rapport au questionnement débattu, le positionnement des débatteurs pouvant être pour ou contre les autres points de vue, à moins qu'ils se situent dans un positionnement neutre d'examen des différents points de vue; par rapport aux autres participants qui défendent un point de vue similaire ou différent, les rapports étant alors de complémentarité ou antagonistes.

- La situation de communication comme dispositif d'échange - L'échange langagier se déroule dans un espace à l'intérieur duquel les participants sont disposés selon un certain dispositif, lequel oriente la circulation de leur parole. Cet espace peut être ouvert ou fermé. Lorsqu'il est ouvert, le dispositif est celui d'une scène publique, et la parole qui circule entre les participants est en plus diffusée en dehors de celui-ci. Ainsi les protagonistes savent que, au-delà de leur interaction, ils s'adressent à un auditoire, présent ou absent, parfois véritable destinataire des propos de chacun d'eux. Lorsque l'espace est fermé, le dispositif est celui d'une scène privée, et la parole circule de façon biunivoque impliquant les protagonistes dans des relations interpersonnelles, sans tenir compte, en principe, de ce qui peut se passer en dehors de cette scène. 


\section{UNE TYPOLOGIE DES ÉCHANGES}

Selon ces critères, on peut établir une typologie des échanges autour de trois catégories de base que sont les échanges de coopération, les échanges de confrontation et les échanges d'affrontement.

- Échanges de coopération - Dans ce type d'échange, les protagonistes ne sont pas placés en relation antagonique. Leurs échangent se complètent par la demande ou la transmission d'information, apportent des explications, affirment des opinions. On y trouve divers genres: la conversation qui se caractérise par une prise de parole libre entre des participants qui estiment qu'ils se trouvent dans des rapports d'égalité, dont les rôles discursifs sont divers (questionner, répondre, affirmer, critiquer, objecter, acquiescer), sans qu'ils soient tenus de se centrer sur un seul questionnement, chacun pouvant s'autoriser à en changer, et de plus ils peuvent se permettre de s'exprimer de façon ludique (jeux de mots, blagues, bon mots, etc.); l'interview qui se caractérise par un échange de questions-réponses, l'interviewer cherchant à obtenir certaines informations, l'interviewé répondant de façon directe ou détournée, selon les enjeux de l'échange, l'interview journaliste n'ayant pas la même finalité que l'interview d'enquête scientifique; la simple demande d'information, lorsqu'une personne s'adresse à un passant dans la rue, ou à une officine d'information (guichet, comptoir, centre d'accueil des clients, etc.), pour obtenir un renseignement, elle se trouve alors dans la situation d'avoir à solliciter son interlocuteur, et ce dernier, par convention sociale ou de par son emploi, se trouve dans la situation de devoir répondre.

- Échanges de confrontation - L'échange de confrontation établit, contrairement aux précédents, un rapport antagonique entre les interlocuteurs, à des degrés divers. Vu l'enjeu de l'échange, ils sont conduits à s'opposer les uns aux autres, à contredire le point de vue de l'autre et à défendre le sien propre. Mais cet antagonisme se produit autour d'un même questionnement, car c'est par rapport à celui-ci que se défendent les positionnements. L'échange de confrontation est un échange polarisé qui se caractérise par une circulation des paroles thématiquement centrées, et un rapport d'opposition entre des positionnements pour (défendre) ou contre (critiquer), créant du dissensus, mais pouvant aboutir à des accords. C'est dans ce cadre que l'on trouve les genres, diversement nommés, discussion, débat et controverse. On n'entrera pas ici dans le détail des caractéristiques de chacun d'eux ${ }^{2}$,

2 Pour plus de précisions sur ces catégories, on consultera Charaudeau 2017, chap. I. 
puisque sera ensuite définit ce qui fait l'objet de cet article, à savoir: la controverse.

- Échanges d'affrontement - Dans l'échange d'affrontement, le rapport entre les interlocuteurs est a priori antagonique, mais en plus il n'y a pas de recherche d'accord, chacun défendant son point de vue sans se préoccuper de celui de l'autre. Le malentendu est permanent en raison de l'absence d'un terrain commun de questionnement, et l'échange n'aboutit ni à un accord, ni à un consensus, ni à un changement d'opinion, chacun campan sur ses positions. L'antagonisme est d'autant plus fort que, la plupart du temps, c'est l'autre qui est directement attaqué, davantage en tant que personne (ad personam) que pour ses idées. Ce genre d'échange est appelé tantôt dispute, tantôt querelle, mais il s'agit toujours d'un rapport polémique, ce que nous allons voir plus loin.

\section{LA CONTROVERSE}

On définira la controverse en utilisant les critères ci-dessus décrits.

Le statut des protagonistes est d'égalité ou de différence mais avec un égal droit à la prise de parole, et celle-ci, selon les dispositifs, est plus ou moins réglée. Les participants parlent toujours au nom d'un savoir de connaissance ou de croyance, en témoignant d'un avis personnel ou de l'opinion d'un groupe.

La thématique est polarisée, c'est-à-dire qu'elle fait l'objet d'un questionnement, ce qui en fait sa condition de "disputabilité". En effet, pour qu'il y ait argumentation et contre-argumentation, il faut que le thème débattu fasse problème, autrement dit qu'il soit lui-même divisé en, au moins, deux vérités ou opinions possibles, de sorte que les débatteurs puissent défendre l'une d'entre elles et réfuter l'autre. Que soit discutée une question scientifique (la mémoire de l'eau), une question sociétale (la violence), ou politique (la laïcité), la thématique doit être cadrée par une problématique qui ouvre la porte à des positionnements différents.

Le positionnement des participants à la controverse est orienté pour ou contre les termes de la problématique, ce qui instaure une relation antagonique entre eux. Cependant, dans cette relation, ils ont obligation d'argumenter car il s'agit de défendre sa position et de contrecarrer celle de l'autre. Ce qui n'empêche que, au terme d'un certain échange de paroles, se produisent des accords. 
L'espace, c'est-à-dire la scène sur laquelle se déroule la controverse, est plus ou moins ouvert ou fermé. S'il est ouvert, c'est que l'échange entre les participants se fait au regard d'un public-tiers, présent ou absent, en position d'avoir à s'informer, à évaluer les arguments des participants, à prendre parti, ou simplement à se faire une opinion, comme c'est le cas dans les débats télévisés. S'il est fermé, c'est que les participants se reconnaissent un statut égal, une compétence égale, comme on le verra dans la controverse scientifique. Toute controverse a un besoin d'un ancrage institutionnel de mise en visibilité des échanges, dans un dispositif plus ou moins régulateur.

L'enjeu de vérité de la controverse est de parvenir à établir une "vérité", ou d'"avoir raison”, toujours au regard d'un public-tiers, qu'il soit présent ou absent. Ce qui fait que le résultat d'une controverse est imprévisible dans ses effets. Il n'est pas sûr que les participants parviennent à des accords, encore moins à un consensus, et il est encore moins sûr que le public soit en mesure de se faire une opinion. On dira que la controverse est à la fois de l'ordre du "décidable" et de l'“indécidable" ${ }^{3}$.

La controverse correspond bien à la controversia de la rhétorique en ce que, contrairement à la disputatio, elle permet une confrontation argumentée, avec l'espoir de faire accepter, par un public-tiers, une vérité contre une autre.

\subsection{Une typologie des controverses}

Les controverses varient selon les caractéristiques des mêmes critères (statut, thématique, positionnement, enjeu) qui ont permis de classer les types d'échange. Cela permet de distinguer trois types de controverse: la controverse scientifique, la controverse doctrinale et la controverse sociale.

\subsubsection{La controverse scientifique}

Le statut de parole des protagonistes d'une controverse scientifique est d'égalité du point de vue du savoir. Les protagonistes sont par définition des pairs. Qu'on les appelle savants, spécialistes, chercheurs, penseurs, experts, leur statut, au regard des savoirs de connaissance, leur donne un égal droit à la parole: c'est là leur légitimité. Ils peuvent appartenir à des disciplines différentes, mais ils sont considérés comme des interlocuteurs

3 Et ce, contrairement à Marcelo Dascal (1995) pour qui la controverse “n'est ni décidable comme la discussion, ni indécidable (rationnellement) comme la dispute”. 
ayant même légitimité de parole au regard de leur spécialité. Ainsi d'une controverse sur la notion de race ayant réuni des biologistes, des juristes, de sociologues et des linguistes.

La thématique d'une controverse scientifique mobilise des savoirs de connaissance, les spécialistes visant à établir une vérité indépendamment de leur subjectivité. Est mise en œuvre une raison savante, dont les explications s'appuient sur des procédures d'observation, d'expérimentation, de calcul et/ou de spéculation logique, explications qui peuvent être vérifiées et utilisés par toute autre personne ayant même compétence. Ce qui explique que le sujet parlant adopte un positionnement si se veut idéalement neutre, sans jugement personnel, tendant à l'objectivité, et disparaissant derrière un énonciateur qui représenterait "la pensée scientifique". Cependant, comme pour qu'une thématique soit objet de controverse il faut qu'elle soit problématisée, les pairs, participant au débat, auront à prendre des positions (parfois appelées "thèses") pour ou contre les termes du questionnement. Par exemple, à propos de problèmes relatifs au réchauffement climatique s'est discutée la question de savoir si les causes sont dues aux comportements des hommes, ou aux grands cycles de la nature.

L'espace, dans lequel se déroules les controverses scientifiques, est plus ou moins institutionnel, plus ou moins académique (colloque scientifique, forum de savants, commission d'experts, séminaire de recherche, revue scientifique), mais en tout état de cause il est fermé, ce qui n'empêche pas qu'il en soit donné un écho en dehors de celui-ci. Le dispositif qui le structure est plus ou moins contraignant, plus ou moins régulé, et peut s'inscrire dans divers supports: les interventions d'un colloque, paraissent ensuite dans un ouvrage collectif ou dans une revue.

L'enjeu de la controverse scientifique est donc toujours, d'une manière ou d'une autre, un enjeu de vérité. Et le discours qui soutient cette vérité est de l'ordre de la démonstration. Évidemment, il s'agit d'une vérité qui sera discutée, dont certains aspects seront mis en cause, et qui pourra apparaître, au terme de la discussion, fragmentée, ce qui fait qu'au bout du compte, aussi bien pour les participants que pour ceux qui en auront connaissance, la controverse leur offrira la possibilité, soit d'adhérer à l'une des thèses (ordre du décidable), soit de s'en tenir à une position d'expectative (ordre de l'indécidable).

\subsubsection{La controverse doctrinale}

Comme dans la controverse scientifique, les participants à une controverse doctrinale ont un statut de parole égalitaire, du point de vue du 
savoir concernant la doctrine à propos de laquelle ils débattent, savoir dont ils sont censés partager la connaissance. Qu'ils soient théologiens, prêtres, évêques, pasteurs, rabbins, imams, les participants ce sont des pairs. C'est lorsque surgit une contestation sur un point de dogme, qu'ils sont conduits à se positionner pour ou contre les différents points de vue qui s'expriment, chacun, au nom du savoir révélé qui les autorise à interpréter les textes sacrés. Mais à la différence des pairs de la controverse scientifique qui parlent au nom de la science, les débatteurs de la controverse doctrinale parlent au nom d'un savoir exégétique inspiré par une force divine.

La thématique est également spécialisée, mais elle concerne ici un point de dogme qui touche une croyance révélée. Le savoir n'est pas ici de connaissance scientifique, mais de révélation. Dans les deux cas, la vérité se trouve dans un lieu extérieur au sujet, mais cette vérité de révélation n'a pas à être prouvée ni vérifiée, et elle n'est jamais mise en cause. Ce qui est discuté ce sont les termes de textes qui témoignent de cette vérité et dont certains points font dissensus quant à leur interprétation. Ainsi en fut-il à propos de la Sainte Trinité qui a occupé les théologiens durant cent vingt-cinq années, sur le questionnement de savoir si le Père, le Fils et le Saint-Esprit pouvaient être une même substance, jusqu'au grand débat du Concile de Nicée en 451.

L'espace est lui aussi fermé dans un cadre institutionnel, lieu symbolique qui témoigne de son caractère sacré. En cela la scène de la controverse est encore plus fermée que celle de la controverse scientifique, car elle doit porter la marque de ce caractère sacré qui se manifeste, à la fois, par le lieu, la qualité de l'Assemblée et la présence d'une autorité religieuse (Église, Conclave, Consistoire, Synode, Concile, Conférence théologique).

L'enjeu d'une telle controverse est donc d'engagement pour la défense d'une vérité révélée dont on doit pouvoir dire: “C'est dans la doctrine, c'est ainsi qu'il faut l'interpréter". Cet enjeu produit un discours exégétique, une herméneutique, un travail d'interprétation destiné à défendre les écrits sacrés, ce qui exclue toute possibilité de critique des textes, qu'il ne faut jamais toucher, car il s'agit toujours in fine de les sauver.

On peut étendre la controverse doctrinale aux cas où le savoir, pour être de connaissance scientifique, se transmue, non pas en savoir de révélation, mais en savoir dogmatique. Un savoir qui se referme sur luimême, non point comme une théorie "jusqu'à preuve du contraire", mais comme une théorie qui ne souffre pas de remise en cause de ses fondements. Dès lors, les textes qui constituent ces fondements jouent, eux aussi, un rôle de référence absolue jusqu'à revêtir un caractère sacré. Là 
aussi, il faut "sauver" à tout prix l'intégrité immuable d'une pensée, et les débatteurs s'érigent en défenseurs de théories, comme les débatteurs théologiens. Les textes qui fondent ces théories deviennent alors des objets intangibles, tiennent lieu de dogme, et leurs défenseurs s'érigent en gardiens du Temple. On a vu se produire ce phénomène dans le domaine philosophique entre les marxistes, les léninistes et les trotskistes, autour de l'interprétation des écrits de Marx; dans le domaine de l'économie, entre partisans de Friedman et partisans de Keynes; dans le domaine de la psychanalyse, entre partisans de Freud, de Jung, de Lacan ${ }^{4}$.

\subsubsection{La controverse sociale}

Les statuts de parole des participants à une controverse sociale, au contraire des cas précédents, sont différents et ont des références de savoir différentes. Ils jouissent cependant d'une égalité de statut de parole dans le droit à s'exprimer du fait qu'ils parlent au nom du groupe qu'ils représentent (religieux, politique, syndical, associatif, citoyen), dont ils sont les porte-parole et qui en retour les légitime. Mais dans la controverse sociale, les interlocuteurs ne sont pas nécessairement des pairs. Ils n'ont pas le même statut social ni les mêmes références de savoir. De plus, ce savoir est relatif à leur personne ou au groupe qu'ils représentent. C'est un savoir subjectif qui dit: "Moi, je pense que...", et non point: "La science dit que...", ou "La doctrine dit que...". Du même coup, leur positionnement se fait au nom de valeurs morales. On verra cependant que ces savoirs tendent à se mélanger, voire se donnent les uns pour les autres.

La thématique n'est pas en son principe spécialisée. Elle concerne des questions de société, et les savoirs auxquels il est fait référence sont des savoirs d'opinion. Ces savoirs sont des savoirs de croyance, non pas au sens des savoirs de révélation, mais au sens de savoirs que partagent les individus vivant en société. Ils ne s'apparentent pas non plus à des savoirs de connaissance qui procéderaient d'analyses scientifiques, car ils procèdent du regard que le sujet porte sur l'empirie du monde dans lequel il est plongé et dont il tire un savoir d'expérience. Il s'agit donc d'un savoir qui dépend de la subjectivité du sujet, toujours empreint de jugement commun. Le savoir d'opinion n'a d'autre garde fou que celui de l'expérience répétitive et du consensus.

L'espace des controverses sociales, différemment des cas précédents, est toujours ouvert et plus ou moins institutionnalisé. L'espace est dit

4 Voir à ce propos Moscovici 1961. 
ouvert dans la mesure où la portée des échanges est publique ou susceptible d'être diffusée publiquement. Alors que les controverses précédentes étaient fermées sur elles-mêmes du fait que le savoir ne pouvait être partagé que par des spécialistes, celles-ci se déroulent avec des participants à statuts divers, porteurs de savoirs qui circulent dans l'espace public. De plus, les lieux de ces controverses sont eux-mêmes divers, certains ayant un caractère institutionnel (tribunaux de justice), d'autres faisant l'objet d'une organisation ritualisée (médias, réseaux sociaux), d'autres encore, moins formellement organisés, mais rassemblant des individus dans le but déterminé de traiter certaines questions d'intérêt général (groupements militants, associations, assemblées citoyennes).

L'enjeu de la controverse sociale n'est donc pas de vérité mais de défense de valeurs afin de faire entendre un point de vue qui se voudrait témoin d'un engagement moral. L'apparition d'un thème qui concerne l'ensemble de la société, et qui d'une façon ou d'une autre heurte la conscience morale ou le sentiment d'injustice, voire provoque de l'indignation empreinte d'émotion, provoque l'intervention des représentants de divers secteurs sociaux voulant défendre une certaine position. De ce fait, la controverse sociale ne permet pas l'établissement d'un cadre commun d'évaluation à propos duquel il serait possible de trancher par consensus. Le lieu discursif n'est pas de décidabilité mais de proposition d'une opinion. Dans la controverse scientifique, il est question de vérité dite objective, dans la controverse doctrinale de vérité de révélation, et dans la controverse sociale il est question de vérité subjective, qui s'énonce en jugement de conviction. Et donc le discours tenu par les participants à ce type de controverse n'est pas tant de démonstration que de persuasion de l'auditoire.

On verra les différences entre ces types de controverse dans la $\mathrm{Fi}$ gure 1 ci-dessous.

Chacune de ces catégories est définie de façon idéale. Chacune constitue un idéal-type comme l'a défini Max Weber ${ }^{5}$. Il s'agit de la typification d'un comportement langagier qui résulte de l'observation des points communs et des variétés qui caractérisent a minima les différentes réalisations de ces comportements. Mais dans le fonctionnement ordinaire du langage, on ne trouve pas toujours réalisée la catégorie à l'état pur. Par exemple, controverses scientifiques et controverses doctrinales se définissent de façon distincte, mais comme on l'a vu dans la proposition d'extension de cette dernière à des domaines non religieux (psychanalyse, économie), une controverse scientifique peut dériver en controverse doctrinale.

5 Voir à ce propos Weber 2000. 


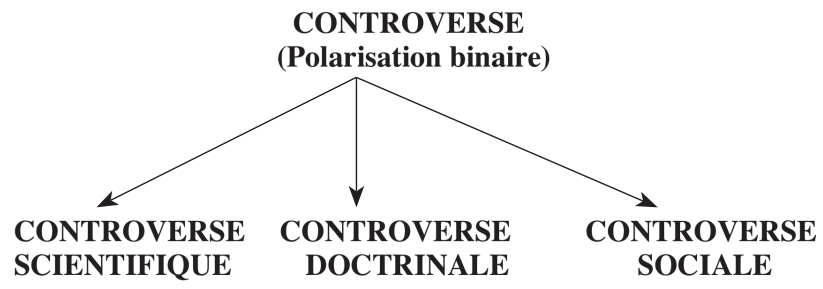

Relation antagonique de confrontation

Thématique polarisée

Positionnement clivé

$\begin{aligned} & \text { égalité des statuts } \\ & \text { (pairs spécialisés) }\end{aligned}$
thématique
(savoir connaissance)
positionnements
(sur théorie)
un seul espace
(fermé)
Enjeu de "vérité"
(démonstration)
(décidable/indécidable)

égalité des statuts

(pairs doctrine)

statuts divers

(représentants)

\author{
thématique \\ (savoir doctrinal)
}

positionnements clivés

(sur doctrine)

un seul espace
(fermé)

Enjeu de "défense"

(interprétation)

(décidable)

\section{n. thématiques}

(savoir d'opinion)

positionnements clivés

(sur valeurs)

plusieurs espaces

(ouverts)

\section{Enjeu d'engagement \\ (moral-citoyen) \\ (indécidable)}

Figure 1. - La controverse.

De même, une question pouvant faire l'objet d'une controverse scientifique, comme les questions de bioéthique (différence entre cellules souches et embryon, fin de vie, procréation médicalement assistée), peut se transformer en controverse sociale dans la mesure où interviennent des acteurs représentant des groupes sociaux différents (le corps médical, les confessions religieuses, les associations familiales), la question n'étant plus traitée d'un point de vue technique mais d'un point de vue moral, savoirs de connaissance et savoirs de croyance se mélangeant.

Mais ce mélange, s'il est préjudiciable pour la compréhension des termes du débat, peut avoir aussi une double vertu: celle d'éveiller la population à des problèmes de société qu'elle ignorait ou dont elle ne percevait pas les enjeux; celle de réinterroger la science pour qu'elle continue ses investigations. On l'a vu avec la controverse sur l'utilisation des organismes génétiquement modifiés (OGM), qui comporte un aspect 
économique pour ce qui est du rendement des espèces cultivées entraînant une guerre commerciale, un aspect politico-juridique pour ce qui est de sa réglementation, un aspect éthique pour ce qui est de la responsabilité des gouvernements au regard d'une politique de santé, et qui ne cesse d'interroger la science pour qu'elle arrive à déterminer le degré de nocivité de ces organismes et éventuellement leurs modalités d'application.

Il est aussi des controverses qui naissent dans l'espace public du fait de certains événements, avec l'intervention de différents acteurs sociaux, obligeant le monde scientifique à s'emparer du problème. Ainsi en est-il de la question de la "laïcité" surgie à la suite de l'expulsion de jeunes filles musulmanes voilées du Lycée de Creil, en 1989, qui a tout de suite provoqué une controverse sociale sur l'opportunité de légiférer en la matière, puis une controverse technique entre juristes sur l'interprétation de la loi de 1905, et même une controverse doctrinale à l'intérieur des églises.

\section{De la POlÉmique}

On ne peut parler de débat sans évoquer cette autre catégorie qui semble lui faire concurrence: la polémique. J'ai longuement analysé cette notion dans le chapitre IV de mon ouvrage sur le débat public (2017), et me contenterai d'en souligner les points principaux, car il est nécessaire de ne pas confondre ces deux catégories que sont la controverse et la polémique. Les confondre, comme le font la plupart des écrits, c'est brouiller l'intelligibilité de ce qu'est le débat social.

On ne reviendra pas sur les différentes définitions donnant lieu à distinction entre le polémique, et la polémique, mais on rappellera que cette notion peut être définie, comme en philosophie et en littérature, avec un sens général: "les multiples manifestations textuelles de l'adversité" (Charaudeau et Maingueneau 2002, 437), ou un sens restreint comme procédé visant à disqualifier l'opposant, considéré comme adversaire (Ducrot 1972). Autrement dit, la polémique entendue comme la disputatio des rhétoriciens de l'Antiquité, ou comme la dispute dans le sens moderne.

Pour ce qui me concerne, je propose de considérer la polémique dans son sens restreint, comme le propose Catherine Kerbrat-Orecchioni, pour qui "Le discours polémique est un discours disqualifiant, c'est-àdire qu'il attaque une cible, et qu'il met au service de cette visée pragmatique dominante $[. .$.$] tout l'arsenal de ses procédés rhétoriques et argu-$ mentatifs" (1980,13). Autrement dit, le discours polémique, dénommée 
ici la polémique, est envisagé comme un ensemble de procédés d'attaque/ défense qui ne peuvent déboucher sur aucun consensus et qui font que la discussion est sans issue possible. Ce qui ne veut pas dire que toute accusation, toute revendication, toute mise en contradiction soit nécessairement polémique. Ces mouvements discursifs sont propres aux rapports de confrontation qui caractérisent certaines discussions et controverses. C'est lorsque la confrontation se transforme en affrontement que l'on verse dans la polémique.

La polémique n'est pas pour autant un genre. A preuve qu'elle peut s'immiscer dans différents genres: elle peut apparaître dans certains moments d'une conversation, d'une discussion sérieuse, d'un débat politique, et même d'une controverse doctrinale ou scientifique, bien que cela ne soit pas conforme aux données du genre. En revanche, elle est de plein droit dans la dispute. Ce qui veut dire que la polémique est de l'ordre de la stratégie discursive. Et comme toute stratégie de discours, elle n'est ni obligatoire ni prévisible. Elle procède de la libre manœuvre ou de la pulsion du sujet polémiquant.

Le moment polémique est, dans l'échange, un coup de force que l'on impose à l'autre, une exigence d'être cru, un croire qui ne peut être cru autrement, ni réfuté, et qui, en plus, met en cause l'interlocuteur. Elle se présente comme un jugement péremptoire qui essentialise le contenu de son jugement, ce qui fait que dans l'instant qu'est énoncé ce jugement, accusatoire à l'endroit de l'interlocuteur, il devient parole de vérité irréductible, et l'interlocuteur se trouve assigné à contre-attaquer de même ou à s'arrêter. Et si d'aventure l'échange se poursuit, ce n'est pas en tant qu' instrument de vérité" (Plantin 2003, 383), comme le souligne Christian Plantin. Il n'y a donc pas d'argumentation, et même on peut dire que toute tentative d'argumentation de la part de l'interlocuteur se trouve bloquée par l'attaque ad bominem ou ad personam du polémiquant. Il s'agit donc véritablement d'un cas de dispute, comme on l'entend ordinairement, entre des personnes qui ne peuvent ni s'entendre, ni se comprendre, ni s'expliquer, et l'on est en totale indécidabilité.

On voit la différence entre controverse et polémique. La première s'inscrit dans un questionnement, et les débatteurs sont amenés à discuter la validité des propositions énoncées; la seconde essaie de déplacer le questionnement, et en tout cas les débateurs ne discutent pas la validité des propositions. La controverse se situe dans un enjeu de vérité, ce qui conduit les débatteurs à argumenter; la polémique se situe dans un enjeu d'avoir raison, et d'avoir raison contre l'autre, ce qui place les débateurs hors argumentation. Enfin, comme on l'a dit, la controverse respecte la contrat d'échange qui en fait un genre, lequel se caractérise par une dé- 
cidabilité possible; la polémique n'est qu'une stratégie individuelle qui provoque le blocage de l'argumentation et rend l'échange "indécidable" (Plantin 2003, 383).

\section{LE RÔLE DES CONTROVERSES}

Les controverses, avec leurs caractéristiques problématisantes et argumentatives, devraient permettre d'informer le public sur l'opportunité et les objectifs de projets de société, de l'éclairer sur les enjeux de vérité des grandes questions sociétales qui interpellent les populations et interrogent les valeurs civilisationnelles, et ce dans l'espoir de donner davantage de pouvoir délibératif, voire décisionnel, aux individus voulant vivre ensemble. Mais la tâche n'est pas aisée parce que les rôles que peuvent jouer les controverses dépend des différentes modalités d'organisation du débat public que l'on a décrites: la manière dont son discutées les questions scientifiques et sociales; le pouvoir des acteurs qui y participent; le dispositif socio-communicationnel dans lequel elles se déploient. C'est pourquoi il est nécessaire de distinguer, comme on l'a fait, les différents types de controverse (scientifique, doctrinale et sociale), parce que les régimes et enjeux de vérité ne sont pas les mêmes, et nécessaire de ne pas confondre, pour ce qui est de l'intelligibilité du débat, polémique et controverse. Quant aux controverses sociales, il faut partir du principe que, quels que soient leurs défauts, elles sont toujours utiles dans une société démocratique.

On sait, depuis Aristote, que, dans un débat public, il y a trois moments: le moment du déclenchement, par un événement qui pose un problème à la société; le moment de l'échange entre divers acteurs sociaux qui prendra, selon les circonstances, forme de controverse ou de polémique; le moment de la délibération qui devrait aboutir à une prise de décision. Mais les effets du débat, c'est-à-dire les retombées sur l'opinion et les décisions ne sont ni immédiates ni uniques. On peut observer, cependant, que lorsque le débat prend allure de controverse, celle-ci est susceptible de jouer trois rôles: un rôle d'alerte, un rôle d'influence, un rôle de révélateur.

Un rôle d'alerte lorsque, à propos d'un événement qui arrive à connaissance du public, est posé un questionnement quant à sa cause, sa raison d'être, sa manière d'être et ses conséquences. Ce n'est pas l'événement en soi qui crée la controverse, mais le fait qu'à son propos surgisse un questionnement qui fera se rencontrer des avis différents ou contraires. 
C'est ce rôle qu'a joué - et que joue - la controverse scientifique sur le réchauffement climatique, opposant les tenants d'une écologie radicale et ceux d'une écologie globale; c'est le rôle que jouent les controverses doctrinales, à l'intérieur de l'Église, à propos de l'interruption volontaire de grossesse, et de la procréation médicalement assistée; c'est le rôle que jouent les controverses sociales concernant l'accueil des immigrés, ou le mariage des homosexuels. La controverse a une fonction de vigilance.

Un rôle d'influence, qui est difficile à déterminer parce qu'il dépend de nombre de facteurs sociaux. Combien de débats restent sans effets, sont oubliés ou détournés par d'habiles politiciens ou chroniqueurs. Ici aussi doivent être distingués les types de controverse. Les controverses scientifiques entre pairs peuvent durer, mais elles sont ponctuées par des moments de vérité qui font avancer la science; les controverses doctrinales, verrouillées qu'elles sont à l'intérieur du cénacle où elles se réalisent, aboutissent à des accords qui sont ensuite publiés, renforçant ainsi la doctrine; les controverses sociales, en revanche, sont ouvertes, et on n'en voit pas toujours les effets. Bien sûr, il y a eu, dans le passé, la controverse à propos de l'affaire Dreyfus, naguère, celle sur l'affaire du sang contaminé, plus récemment, celles sur le Voile à l'école et le mariage pour tous, qui se sont soldées par des décisions concrètes, ce qui ne garantit pas leur clôture définitive. Mais combien d'autres qui restent en suspens et qui ressurgissent sans fin, comme celles sur la lä̈cité et la fin de vie. Il convient donc de s'interroger, à chaque fois, sur les conditions dans lesquelles se sont déroulées les controverses pour en mesurer l'influence, sans préjuger de leurs résultats.

Un rôle révélateur qui concerne le fait que tout débat public témoigne d'un certain état des imaginaires collectifs d'une société, à une époque donnée: les lois de la nature contre les lois de la culture (à propos des questions de bioéthique); les valeurs universalistes contre les valeurs relativistes (à propos des questions identitaires); les positions conservatrices défendant la tradition contre les positions progressistes défendant le changement; les conceptions monoculturalistes des peuples contre les conceptions multiculturalistes; les visions politiques fédéralistes contre les visions nationalistes. On a pu observer, en France, ces oppositions d'imaginaires à propos des débats sur le "Mariage pour tous" et la conception du lien familial; à propos du Voile islamique et la conception de la laïcité; à propos des actes de délinquance et des attentats et le droit des victimes; à propos des rapports entre les sexes, de l'inégalité, du harcèlement et de la violence; à propos des mouvements de révolte contre la précarité et les conditions de vie quotidienne, comme celui de Gilets jaunes. 


\section{Conclusion}

Dans cette prolifération des débats provoqués ou entretenus par le monde politique, les médias et les réseaux sociaux, dans lesquels circulent autant de fausses informations et contre-vérités que des propos argumentés, il est urgent d'apprendre à faire la distinction entre rapports de confrontation et d'affrontements, polémique et controverse, controverse scientifique, doctrinale et sociale. Les controverses ne sont pas le tout de la vie démocratique mais elles y contribuent pour une grande part, si tant est que l'on croit en la nécessité de discuter et de délibérer avant de se faire une opinion et de prendre une décision. Tant bien que mal, elles possèdent une fonction socialisatrice de dialogue, d'intégration, et de reconnaissance de la différence de l'autre.

\section{RÉFÉRENCES BIBLIOGRAPHIQUES}

Charaudeau, Patrick. 2017. Le débat public. Entre controverse et polémique. Enjeu de vérité, enjeu de pouvoir. Paris: Lambert-Lucas.

Charaudeau, Patrick, et Dominique Maingueneau. 2002. Dictionnaire d'analyse du discours. Paris: Seuil.

Dascal, Marcelo. 1995. "Observations sur la dynamique des controverses". Cabiers de linguistique française 17 (Les différents plans d'organisation du dialogue et leurs interrelations. Actes du VI ${ }^{\text {ème }}$ Colloque de pragmatique de Genève, 1517 juin 1995 - $2^{\text {ème }}$ partie): 99-121. Genève: Université de Genève.

Ducrot, Oswald. 1972. "De Saussure à la philosophie du langage". Préface à Les Actes de langage, édité par John R. Searle, 11-12. Paris: Hermann.

Kerbrat-Orecchioni, Catherine. 1980. "La polémique et ses définitions". Dans Le discours polémique, édité par Nadine Gelas et Catherine Kerbrat-Orecchioni, 3-40. Lyon: Presses Universitaires de Lyon.

Moscovici, Serge. 1961. La psychanalyse, son image et son public. Paris: Presses Universitaires de France.

Plantin, Christian. 2003. "Des polémistes aux polémiqueurs". Dans La parole polémique, édité par Michel Murat, Gilles Declercq, et Jacqueline Dangel, 377-408. Paris: Champion.

Weber, Max. 2000. Essais sur la théorie de la science. Lyon: Decitre (Pocket-Agora). 